\title{
Time-dependent hydrogen ionisation in 3D simulations of the solar chromosphere
}

\section{Methods and first results}

\author{
J. Leenaarts ${ }^{1,2}$ and S. Wedemeyer-Böhm ${ }^{3}$ \\ 1 Sterrekundig Instituut, Utrecht University, Postbus 80 000, 3508 TA Utrecht, The Netherlands \\ e-mail: j.leenaarts@astro.uu.nl \\ 2 Institute of Theoretical Astrophysics, University of Oslo, PO Box 1029, Blindern, 0315 Oslo, Norway \\ 3 Kiepenheuer Institut für Sonnenphysik, Schöneckstrasse 6, 79104 Freiburg, Germany \\ Received 27 July 2006 / Accepted 16 August 2006
}

\section{ABSTRACT}

Context. The hydrogen ionisation degree deviates substantially from statistical equilibrium under the conditions of the solar chromosphere. A realistic description of this atmospheric layer thus must account for time-dependent non-equilibrium effects.

Aims. Advancing the realism of numerical simulations of the solar chromosphere by improved numerical treatment of the relevant physics will provide more realistic models that are essential for interpretation of existing and future observations.

Methods. An approximate method for solving the rate equations for the hydrogen populations was extended and implemented in the three-dimensional radiation (magneto-)hydrodynamics code $\mathrm{CO}^{5} \mathrm{BOLD}$. The method is based on a model atom with six energy levels and fixed radiative rates. It has been tested extensively in one-dimensional simulations. The extended method has been used to create a three-dimensional model that extends from the upper convection zone to the chromosphere.

Results. The ionisation degree of hydrogen in our time-dependent simulation is comparable to the corresponding equilibrium value up to $500 \mathrm{~km}$ above optical depth unity. Above this height, the non-equilibrium ionisation degree is fairly constant over time and space, and tends to be at a value set by hot propagating shock waves. The hydrogen level populations and electron density are much more constant than the corresponding values for statistical equilibrium, too. In contrast, the equilibrium ionisation degree varies by more than 20 orders of magnitude between hot, shocked regions and cool, non-shocked regions.

Conclusions. The simulation shows for the first time in 3D that the chromospheric hydrogen ionisation degree and electron density cannot be calculated in equilibrium. Our simulation can provide realistic values of those quantities for detailed radiative transfer computations.

Key words. Sun: chromosphere - radiative transfer

\section{Introduction}

Hydrogen is the most abundant constituent of the solar gas and the major electron donor in the solar chromosphere. The assumption of local thermal equilibrium (LTE) for hydrogen atoms is not valid in the chromosphere because the radiative transition rates dominate over the collisional rates. Even the assumption of instantaneous statistical equilibrium, where the level populations of each species at all times are in equilibrium as determined by the local thermodynamic state and the non-local radiation field, is not valid in the dynamical chromosphere. The timescale on which the hydrogen level populations adjust to changes in the atmosphere (which is set by the transition rates between levels) is too long compared to the timescale on which the atmosphere changes. In order to properly model this behaviour one has to solve the rate equations for the populations of all relevant energy levels. We henceforth refer to this approach as time-dependent non-LTE modelling (TD-NLTE).

Kneer (1980) showed that the relaxation timescale for the ionisation of hydrogen varies from $100 \mathrm{~s}$ to $1000 \mathrm{~s}$ in the middle to upper chromosphere. In the nineties, time-dependent hydrogen ionisation was first implemented in one-dimensional (1D) hydrodynamics simulations of the solar chromosphere and corona (Carlsson \& Stein 1992; Hansteen 1993; Carlsson \& Stein 2002). Carlsson \& Stein (2002, hereafter CS2002) gave a detailed analysis of dynamic hydrogen ionisation in 1D, where they included a transition region and a corona in their model. More recently, Rammacher \& Ulmschneider (2003) also implemented a time-dependent treatment of magnesium in a 1D simulation.

The next step is obviously the inclusion of time-dependent hydrogen ionisation into $2 \mathrm{D}$ and $3 \mathrm{D}$ simulations. For reasons of numerical stability and current computer limitations, a full timedependent NLTE treatment of hydrogen in the solar atmosphere is not yet possible in two- and three-dimensional models. In the 1D case a simplified treatment using fixed radiative rates gives a good approximation to the full solution (Sollum 1999). In this paper, we report on the implementation of this method in the $2 \mathrm{D} / 3 \mathrm{D}$ radiation hydrodynamics code $\mathrm{CO}^{5} \mathrm{BOLD}$ code (Freytag et al. 2002). It yields electron densities that are more realistic than in the original simulation, assuming LTE. The electron densities can now directly be used for a more realistic synthesis of chromospheric spectral lines and continua, producing much more reliable results compared to earlier calculations based on instantaneous statistical equilibrium.

We give details on hydrogen ionisation in Sect. 2 and the numerical method in Sect. 3. The results of our numerical simulation are described in Sect. 4 and finally discussed in Sect. 5.

\section{Hydrogen ionisation in the solar chromosphere}

In this section we recapitulate the details of chromospheric hydrogen ionisation as found by CS2002. 
Statistical equilibrium does not hold under chromospheric conditions because the dynamical time scale of the chromosphere is much shorter than the relaxation timescale of hydrogen level populations. The timescale in cool inter-shock regions was found to be of the order of $10^{4} \mathrm{~s}$. Within shocks the relaxation timescale is typically $50 \mathrm{~s}$, owing to the higher temperatures and densities. This is still sufficiently long to prevent equilibration of the hydrogen populations within a shock. Thus, the timedependent rate equations need to be solved to obtain the correct hydrogen level populations. Up to just below the transition region Lyman $\alpha$ may be regarded as being in detailed balance for all practical purposes. Lyman continuum ionisation is negligible. To quote CS2002 literally: "the dominant hydrogen ionization process is photoionization from the second level [...]. There is a very rapid equilibration of the second and all higher levels with the continuum, with a slow collisional leakage of electrons from the ground state to the second level or visa versa, depending on whether hydrogen is ionizing or recombining". Because the ionisation in shocks is much faster than the post-shock recombination, the ionisation degree and electron density tend to represent their shock value, also in the low temperature intershock regions.

\section{Method}

\subsection{The approximate hydrogen radiative transport}

The full time-dependent NLTE radiation transport problem is as yet unsolvable in a 3D (M-)HD code. The demands on memory and processor time are very high, and the currently employed solution methods are not stable enough to handle strong shock waves in a 3D dynamical code. Simplifications are needed in order to compute the non-equilibrium hydrogen ionisation. We therefore adopt the concept of fixed radiative rates in order to avoid computationally expensive lambda iteration for evaluation of mean intensities. The new level populations can be computed from the local gas density and temperature, the imposed radiation field, and the level populations and electron densities from the previous time step. We so follow the example of Sollum (1999) who showed that a simplified treatment of the radiative transport in hydrogen transitions, using fixed radiative rates, gives reasonably accurate results when compared with both static and dynamical calculations in 1D. He compared the full and simplified treatment both in the static case of the VAL3C atmosphere (Vernazza et al. 1981), and in dynamical simulations with the 1D radiation hydrodynamics code RADYN (Carlsson \& Stein 1992). He found that up to $\log \tau_{500}=-5.6$ the differences in the hydrogen level populations between the full and the simplified treatment were at most a few percent. Above this height (just below the transition region in his model) large deviations occur because of the neglect of Lyman transitions in the simplified treatment. Below we will briefly outline his method and our extensions for 2D and 3D simulations. See Sollum (1999) for more details.

\subsubsection{Radiation field}

We assume that the radiation field in each transition, both boundbound and bound-free, can be described by a formal radiation temperature $T_{\text {rad }}$ so that the angle-averaged intensity $J_{v}$ at frequency $v$ is given by

$J_{v}=\frac{2 h v^{3}}{c^{2}} \frac{1}{\mathrm{e}^{h v / k T_{\text {rad }}}-1}$, where $h, c$, and $k$ are Planck's constant, the speed of light, and Boltzmann's constant. The radiation temperature $T_{\text {rad }}$ is different for each transition but independent of frequency within a particular transition. However, $T_{\text {rad }}$ is allowed to vary over position and time. Its value is determined as follows: for each transition the radiation temperature at the upper boundary of the model atmosphere $T_{\text {rad }}^{\text {top }}$ is given as input, as derived by Sollum's detailed 1D study. Then for each time step and each horizontal position we compute $z_{\text {crit }}(x, y)$, the smallest height for which

$J_{v_{0}}\left(T_{\mathrm{e}}\right)=2 J_{v_{0}}\left(T_{\mathrm{rad}}^{\mathrm{top}}\right)$.

Here $T_{\mathrm{e}}$ is the local gas temperature and $v_{0}$ the line centre frequency in the case of bound-bound transition and the ionisation threshold frequency in the case of bound-free transitions. Below this height we set $T_{\text {rad }}=T_{\mathrm{e}}$, i.e., we set the radiation temperature equal to the local gas temperature. Above this height, we set

$J_{v}(z)=B_{v}\left(T_{\text {rad }}^{\mathrm{top}}\right)+\left[B_{v}\left(T_{\mathrm{e}}\left(z_{\text {crit }}\right)\right)-B_{v}\left(T_{\mathrm{rad}}^{\mathrm{top}}\right)\right]\left(\frac{m_{c}(z)}{m_{c}\left(z_{\text {crit }}\right)}\right)^{H}$.

Here $m_{c}(z)$ is the column mass at height $z$ and $H$ is a free parameter that was determined by Sollum. This means we have a decay of $J_{v}$ as a function of column mass. From this $J_{v}(z)$ we calculate $T_{\text {rad }}(z)$ for $z>z_{\text {crit }}$.

This procedure ensures the radiation temperature connects smoothly to the gas temperature in each column at $z_{\text {crit }}$. There may be strong horizontal gradients in the radiation temperature around this height if the gas temperature shows strong horizontal gradients as well. The most important radiative transition for hydrogen ionisation in the chromosphere is the Balmer continuum. In this transition the radiation temperature ranges from $6250 \mathrm{~K}$ at $z_{\text {crit }}$ to $5237 \mathrm{~K}$ at the top of the computational domain.

\subsubsection{Radiative bound-bound rate coefficients}

We restate the derivation of the radiative rate coefficients by Sollum (1999).

For lines, the assumption of a narrow line $\left(J_{v} / v\right.$ is constant over the line profile) gives the following expression for the radiative excitation rate coefficient:

$$
\begin{aligned}
R_{l u} & =4 \pi \int_{0}^{\infty} \frac{\sigma_{l u}(v)}{h v} J_{v} \mathrm{~d} v \\
& =B_{l u} J_{v_{0}} \\
& =\frac{4 \pi^{2} e^{2}}{h v_{0} m_{\mathrm{e}} c} f_{l u} \frac{2 h v_{0}^{3}}{c^{2}} \frac{1}{\mathrm{e}^{h v_{0} / k T_{\mathrm{rad}}}-1}
\end{aligned}
$$

Here $\sigma_{l u}(v)$ is the absorption cross section at frequency $v, B_{l u}$ is the Einstein coefficient for radiative excitation, $J_{v_{0}}$ is the angleaveraged radiation field at the line centre frequency $v_{0}$, and $f_{l u}$ is the oscillator strength. In addition we used the relation

$\int_{0}^{\infty} \sigma_{l u}(v) \mathrm{d} v=\frac{h v_{0}}{4 \pi} B_{l u}=\frac{\pi e^{2}}{m_{\mathrm{e}} c} f_{l u}$.

The bound-bound radiative deexcitation rate coefficient is

$$
\begin{aligned}
R_{u l} & =A_{u l}+B_{u l} J_{v_{0}} \\
& =\frac{g_{l}}{g_{u}} \mathrm{e}^{h v_{0} / k T_{\mathrm{rad}}} R_{l u} .
\end{aligned}
$$

Where $g_{l}$ and $g_{u}$ are the statistical weights of the lower and upper level and $A_{u l}$ and $B_{u l}$ are the Einstein coefficients for spontaneous and stimulated deexcitation. 


\subsubsection{Radiative bound-free rate coefficients}

The hydrogen bound-free excitations have a Kramers absorption cross section

$\sigma_{i c}(v)=\alpha_{0}\left(\frac{v_{0}}{v}\right)^{3}, v>v_{0}$,

where $\alpha_{0}$ is the absorption cross-section at the ionisation edge frequency $v_{0}=\left(E_{c}-E_{i}\right) / h$, where $E_{c}$ and $E_{i}$ are the energy of the continuum and the bound level. In this case the radiative excitation rate coefficient can be written as

$$
\begin{aligned}
R_{i c} & =4 \pi \int_{v_{0}}^{\infty} \frac{\sigma_{i c}(v)}{h v} J_{v} \mathrm{~d} v \\
& =\frac{8 \pi}{c^{2}} \alpha_{0} v_{0}^{3} \int_{v_{0}}^{\infty} \frac{1}{v} \frac{1}{\mathrm{e}^{h v_{0} / k T_{\text {rad }}}-1} \mathrm{~d} v \\
& =\frac{8 \pi}{c^{2}} \alpha_{0} v_{0}^{3} \sum_{n=1}^{\infty} E_{1}\left[n \frac{h v_{0}}{k T_{\text {rad }}}\right],
\end{aligned}
$$

where $E_{1}$ is the first exponential integral and $J_{v}=B_{v}\left(T_{\text {rad }}\right)$. The bound-free radiative deexcitation rate coefficient is

$R_{c i}=\frac{8 \pi}{c^{2}} \alpha_{0} v_{0}^{3}\left[\frac{n_{i}}{n_{c}}\right]_{\mathrm{LTE}} \int_{v_{0}}^{\infty} \frac{\sigma_{i c}(v)}{h v}\left(\frac{2 h v^{3}}{c^{2}}+J_{v}\right) \mathrm{e}^{-h v / k T_{\mathrm{e}}} \mathrm{d} v$.

Using the Kramers cross-section and the radiation temperature this can be written as

$R_{c i}=\frac{8 \pi}{c^{2}} \alpha_{0} v_{0}^{3}\left[\frac{n_{i}}{n_{c}}\right]_{\mathrm{LTE}} \sum_{n=0}^{\infty} E_{1}\left[\left(n \frac{T_{\mathrm{e}}}{T_{\mathrm{rad}}}+1\right) \frac{h v_{0}}{k T_{\mathrm{e}}}\right]$.

Here $n_{i}, n_{c}$, and $T_{\mathrm{e}}$ are the population of the lower level, the population of the continuum, and the local kinetic temperature, respectively.

\subsubsection{Collisional rate coefficients}

We take the collisional rate coefficients $C_{i j}$ from Johnson (1972), who provides approximate cross sections for collisions of hydrogen with electrons.

\subsubsection{Electron densities}

As hydrogen is the most abundant element in the solar gas, ionisation of hydrogen severely affects the electron densities, which in turn enter into the collisional rate coefficients. LTE electron densities are a poor choice at chromospheric heights. Hence, we adopt a hybrid between LTE and TD-NLTE electron densities. The solution of the rate equations in our TD-NLTE approach provides the population density of the continuum level $\mathrm{H}$ II that is at the same time the contribution of hydrogen to the electron number density. To this we add the LTE electron densities from the other elements by solving the Saha equation keeping the hydrogen ionization degree constant. For computational speed, we use a pre-calculated table that gives the electron density as a function of hydrogen ionisation degree, gas temperature, and mass density. Parts of the RH code (Uitenbroek 2001) and partition functions by Kurucz ${ }^{1}$ were used for the creation of the table.

\footnotetext{
${ }^{1}$ http://kurucz.harvard.edu/atoms/PF/
}

\subsubsection{Hydrogen level populations}

The equations and methods described above are used for solving the level population evolution equations for hydrogen,

$\frac{\partial n_{i}}{\partial t}+\nabla \cdot\left(n_{i} \boldsymbol{v}\right)=\sum_{j \neq i}^{n_{l}} n_{j} P_{j i}-n_{i} \sum_{j \neq i}^{n_{l}} P_{i j}$

where $n_{l}, n_{i}$, and $\boldsymbol{v}$ are the total number of levels, the number density of level $i$, and the flow velocity. The second term on the left hand side of Eq. (16) represents changes in the population number due to advection by the hydrodynamic flow. The right hand side terms are rates into and out of level $i$ by collisional and radiative transitions between the energy levels with $P_{j i}$ and $P_{i j}$ being the rate coefficients for transitions from level $j$ to $i$ and vice versa. The rate coefficient is the sum of collisional and radiative rate coefficients:

$P_{i j}=C_{i j}+R_{i j}$.

\subsection{Numerical implementation}

Numerically, Eq. (16) is solved in two steps. In the first step, the populations from the previous time step are advected with the flow in the hydrodynamics computation. In the second step, the advected populations, mass density, and temperatures are used to calculate the new rate coefficients. Then the rate equations (without the advection term) form a system of linear ordinary differential equations of first order, which is solved on the time interval between the previous and current time step using the DVODE package (Brown et al. 1989). The solution yields the level populations for the current time step. This system is solved with a relative accuracy of $10^{-4}$ for each timestep. To ensure exact particle conservation we then scale the updated population densities with the total hydrogen number density, which is directly calculated from the mass density and the mass fraction of hydrogen in the gas. This prevents cumulative buildup of small errors. The code outputs the electron densities and hydrogen level populations for the LTE case and the TD-NLTE case for each grid cell.

\subsubsection{The hydrodynamic simulation}

We performed a three-dimensional numerical simulation using an upgraded version of the $\mathrm{CO}^{5} \mathrm{BOLD}$ code (Freytag et al. 2002). The relaxed end model by Wedemeyer et al. (2004) was adopted as initial model. The computational grid consists of 140 cells in each horizontal direction and 200 cells in the vertical direction. Horizontal resolution is $40 \mathrm{~km}$, vertical resolution ranges from $46 \mathrm{~km}$ at the bottom to $12 \mathrm{~km}$ at the top of the computational domain. The horizontal extent is $5600 \mathrm{~km}$. The vertical extent ranges from $-1300 \mathrm{~km}$ below to $1783 \mathrm{~km}$ above average Rosseland optical depth unity. Radiative transfer is treated in the grey approximation. For more details on the hydrodynamics code see Wedemeyer (2003) and Wedemeyer et al. (2004). We let the simulation advance for $90 \mathrm{~min}$ of solar time. The first $30 \mathrm{~min}$ are reserved to ensure relaxation of the initial conditions, whereas the remaining $60 \mathrm{~min}$ are used for detailed analysis.

\subsubsection{The model atom}

We used a five-level-plus-continuum hydrogen model atom with collisional transitions from each level to each other level. Radiative transitions from the ground level are not allowed, i.e., all Lyman transitions are put in detailed balance. Lyman 


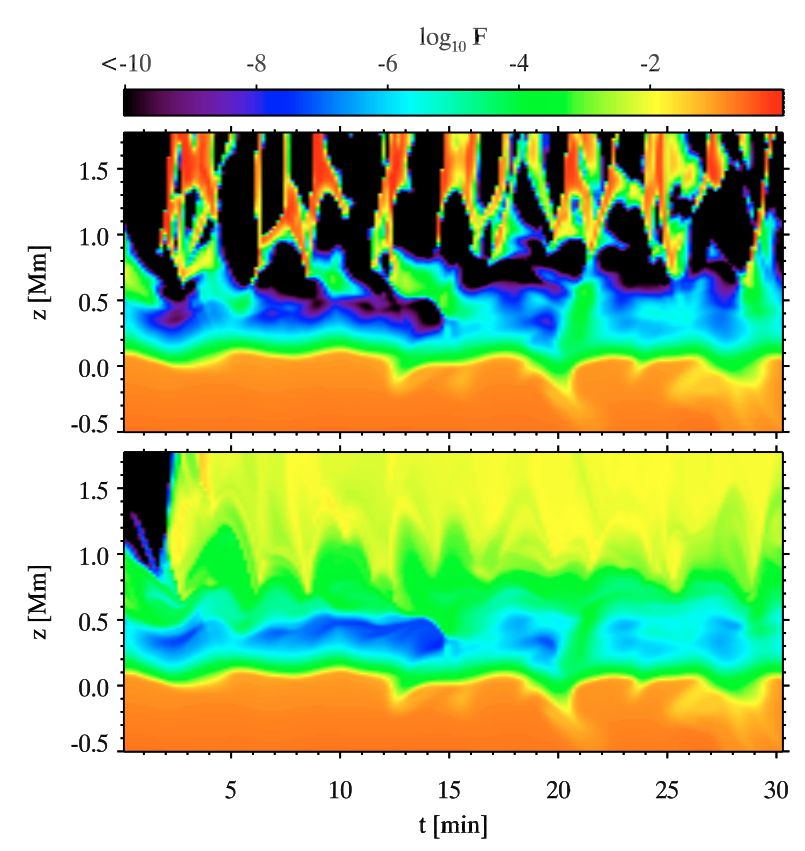

Fig. 1. Time evolution of the ionisation degree $F$ in a column of the simulation for the first $30 \mathrm{~min}$ of the simulation. Upper panel: LTE. Lower panel: TD-NLTE. The TD-NLTE simulation was started with LTE populations. The chromosphere shows large temporal variation in ionisation degree in the LTE case. The ionisation degree in the TDNLTE case is relatively constant after the first $5 \mathrm{~min}$, when the first few shocks have passed.

cooling or heating is thus not possible, but as our model does not include a transition zone no significant effect even in the upper layers of our model is to be expected. The bound-bound oscillator strengths come from Johnson (1972) whereas the bound-free radiative cross sections are from Seaton (1960).

\section{Results}

Figure 1 shows the time evolution of the ionisation degree $F$ along a column in the atmosphere. At $t=0 \mathrm{~min}$, the simulation is started with LTE values for the hydrogen level populations. The LTE panel (upper) shows the chromospheric shocks as red streaks of high ionisation in the upper part of the atmosphere, with cool, neutral gas in between. The typical time between successive shocks is of the order of 2-3 min. In contrast, the ionisation degree in the TD-NLTE case (lower panel) reaches a dynamical equilibrium state of fairly constant ionisation already after $5 \mathrm{~min}$, when the first shocks have passed.

Figure 2 shows horizontal slices through the simulation after $60 \mathrm{~min}$. At $z=0.5 \mathrm{Mm}$ the temperature and density show little structure, as it is in between the reversed granulation layer below (see Leenaarts \& Wedemeyer-Böhm 2005) and the onset of strong shock formation in higher layers. The timedependent ionisation degree is almost in LTE, showing only a somewhat higher ionisation degree in the cooler areas, where over-ionisation in the Balmer continuum occurs. At $z=1 \mathrm{Mm}$ the situation is different. Owing to the steady decline in average density, the waves that are excited by the convective motions in the photosphere have steepened into shocks. Both the temperature and density fluctuations have increased. This has a profound effect on the LTE ionisation degree. Because the ionisation degree is inversely proportional to the electron density, the hydrogen ionisation at equal temperature is much stronger than in deeper layers. In this particular snapshot the peak ionisation degree at $z=1 \mathrm{Mm}$ is 0.89 at $T=6813 \mathrm{~K}$ and the minimum is $3.5 \times 10^{-26}$ at $T=1871 \mathrm{~K}$. In the time-dependent case the ionisation degree has less extreme values, the maximum is 0.019 at $6443 \mathrm{~K}$ and the minimum is $7.8 \times 10^{-5}$ at $2495 \mathrm{~K}$. Note also that the positions of the extrema of the TD-NLTE ionisation degree do not at all coincide with the positions of the LTE extrema. The LTE ionisation degree depends on the local mass density and temperature only, whereas in the time-dependent case it also depends on the previous history of the atmosphere.

At $z=1.5 \mathrm{Mm}$ this effect becomes even more striking. The average density is lower, and temperature and density fluctuations are larger, resulting in even larger LTE ionisation degree fluctuations. In TD-NLTE the transition rates have become so small in the cool, non-shocked areas that there is almost no recombination between the passages of two shock waves. The transition rates are higher in shock fronts as a direct result of higher temperature and density. Although this leads to an increased ionisation degree in shock fronts, it remains small compared to the LTE case. As a result the time-dependent ionisation degree at this height is fairly constant, in this snapshot varying only between 0.0014 and 0.021 with an average of about 0.008 .

Figure 3 shows some physical quantities along a vertical slice of the same snapshot as in Fig. 2. Panel a shows the temperature, with granulation near $0 \mathrm{Mm}$, reversed granulation around $0.2-0.3 \mathrm{Mm}$, and shocks above $\sim 0.7 \mathrm{Mm}$. The mass density (panel b) decreases towards larger height, while its horizontal fluctuations increase. Panels $\mathrm{c}$ and $\mathrm{d}$ show the hydrogen ionisation degree $F$ in LTE and TD-NLTE, respectively. The LTE case follows the increase of horizontal inhomogeneities with height, with high ionisation in the high-temperature shock waves. In contrast, the TD-NLTE case shows only small horizontal variation and the presence of shocks can hardly be discerned. Finally, panels e and f show the electron density in LTE and TD-NLTE. The electron density is mainly set by the hydrogen ionisation and - in first approximation - can be thought of as a multiplication of the density with $F$. Contributions from other elements are dominant only where $F$ is lower than $1 \times 10^{-4}$, i.e., around $0.4 \mathrm{Mm}$ and - in the LTE case - in the cool areas in the chromosphere. Again, in the time-dependent case the extrema and horizontal variations are smaller in TD-NLTE than in LTE.

Figure 4 shows the departure coefficients for each level of our model atom in the same slice as Fig. 3. The departure coefficient is defined as the ratio of the population densities of a particular atomic energy level in NLTE and in LTE, $b_{i}=n_{i} / n_{i}^{\mathrm{LTE}}$. Below $z=0.3 \mathrm{Mm}$ the departure coefficients for all levels are close to 1 , i.e., hydrogen is in or very near LTE. Above these heights deviations occur. The ground level ( $n=1$, lowest panel) is very close to LTE for most of the chromosphere, except in strong shocks. In the shocks the ionisation lags behind compared to LTE, resulting in a slight overpopulation of the ground level (which is the reservoir where ionised hydrogen atoms are coming from). The other levels are strongly collisionally coupled with each other and show similar behaviour in the chromosphere. They are slightly underpopulated in the shock area because the ionisation time scale is comparable or slightly longer than the typical shock crossing time. The density of the ionised state H II (the continuum level) is correspondingly lower than in LTE. Due to the strong coupling to the H II density, $b<1$ for all excited levels of neutral hydrogen $(n=2$ to $n=5)$, too. In the cool intershock areas all levels except $n=1$ are hugely overpopulated. This is because the Saha and Boltzmann equations predict extremely low population fractions for excited states and the continuum for temperatures and electron densities typical for 


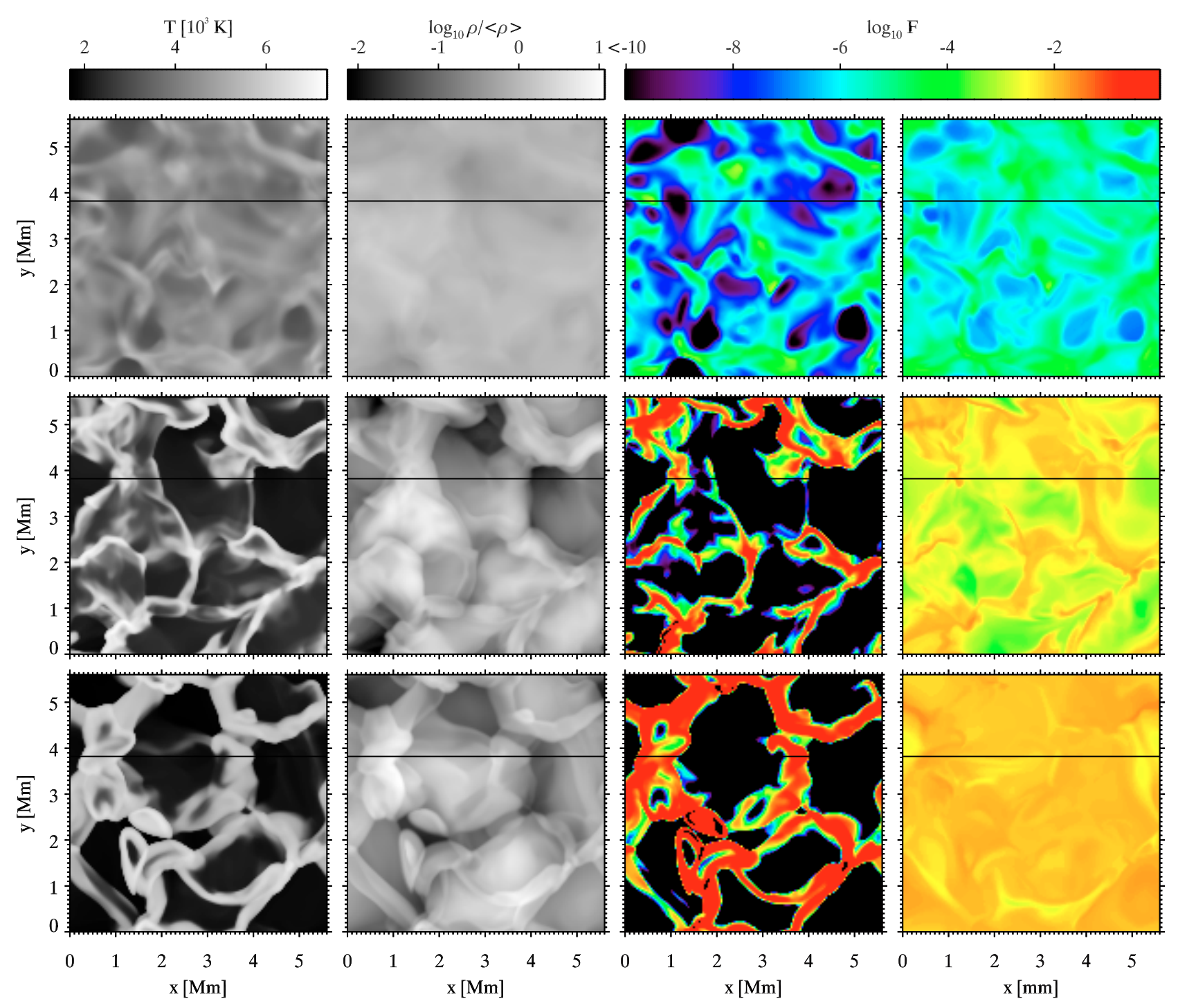

Fig. 2. Horizontal slices through a simulation snapshot at $t=60 \mathrm{~min}$. Columns from left to right: gas temperature, logarithm of the mass density fluctuations $\log (\rho /\langle\rho\rangle)$, LTE ionisation degree and TD-NLTE ionisation degree $F$. Top row: $z=0.5 \mathrm{Mm}$; middle row: $z=1 \mathrm{Mm}$; bottom row: $z=1.5 \mathrm{Mm}$. The LTE and TD-NLTE ionisation degree panels have the same color scale, which has been clipped at log $F=-10$. The black line indicates the position of the vertical cuts of Figs. 3 and 4.

the intershock regions. Thus, these enormous overpopulations simply illustrate the complete failure of Saha-Boltzmann equilibrium partitioning.

Figure 5 shows a height-dependent histogram of the ionisation degree. In LTE there is a strong bifurcation of the ionisation degree as a result of the bimodal temperature distribution in our model chromosphere (cf. Fig. 7 of Wedemeyer et al. 2004). In TD-NLTE this bifurcation is not present. For both cases there is a minimum in average ionisation degree at $z=0.3 \mathrm{Mm}$, roughly corresponding to the classical temperature minimum in 1D static quiet-Sun models. Quite surprisingly, the average ionisation degree in the chromosphere is lower in the TD-NLTE case than in LTE. This is because the average is determined mainly by the high LTE ionisation degree in the shocks whereas in the TD-NLTE case the ionisation degree is smaller there.

\section{Discussion and conclusions}

Figure 6 shows a comparison of the average ionisation degree for our model, the detailed 1D study of CS2002, and the FAL model C (Fontenla et al. 1993). The latter is a static and one-dimensional semi-empirical model, constructed under the assumption of statistical equilibrium, while the RADYN and $\mathrm{CO}^{5} \mathrm{BOLD}$ models are dynamic but differ in the number of spatial dimensions. All models nevertheless show the same qualitative behaviour, with a minimum in ionisation degree between 200 and $500 \mathrm{~km}$, and a rise of the average ionisation degree with height in the chromosphere. The cause of this behaviour, however, is different. In the static FAL-C model, the ionisation degree reflects a corresponding chromospheric temperature rise. In contrast the rise in ionisation degree in the dynamical RADYN and $C^{5}{ }^{5} \mathrm{BOLD}$ models is due to the temperature in shock fronts, which increases with height, without need for a rise in average temperature (Carlsson \& Stein 1995).

Qualitatively our $\mathrm{CO}^{5} \mathrm{BOLD}$ results are comparable to the detailed 1D study of CS2002. We attribute the remaining differences in ionisation degree to the different dimensionality of the code (1D vs. 3D) and the resulting different temperature structure. 1D simulations suffer from shock merging that lead to too high shock temperatures (Ulmschneider et al. 2005). Additionally, we do not include the effect of the 


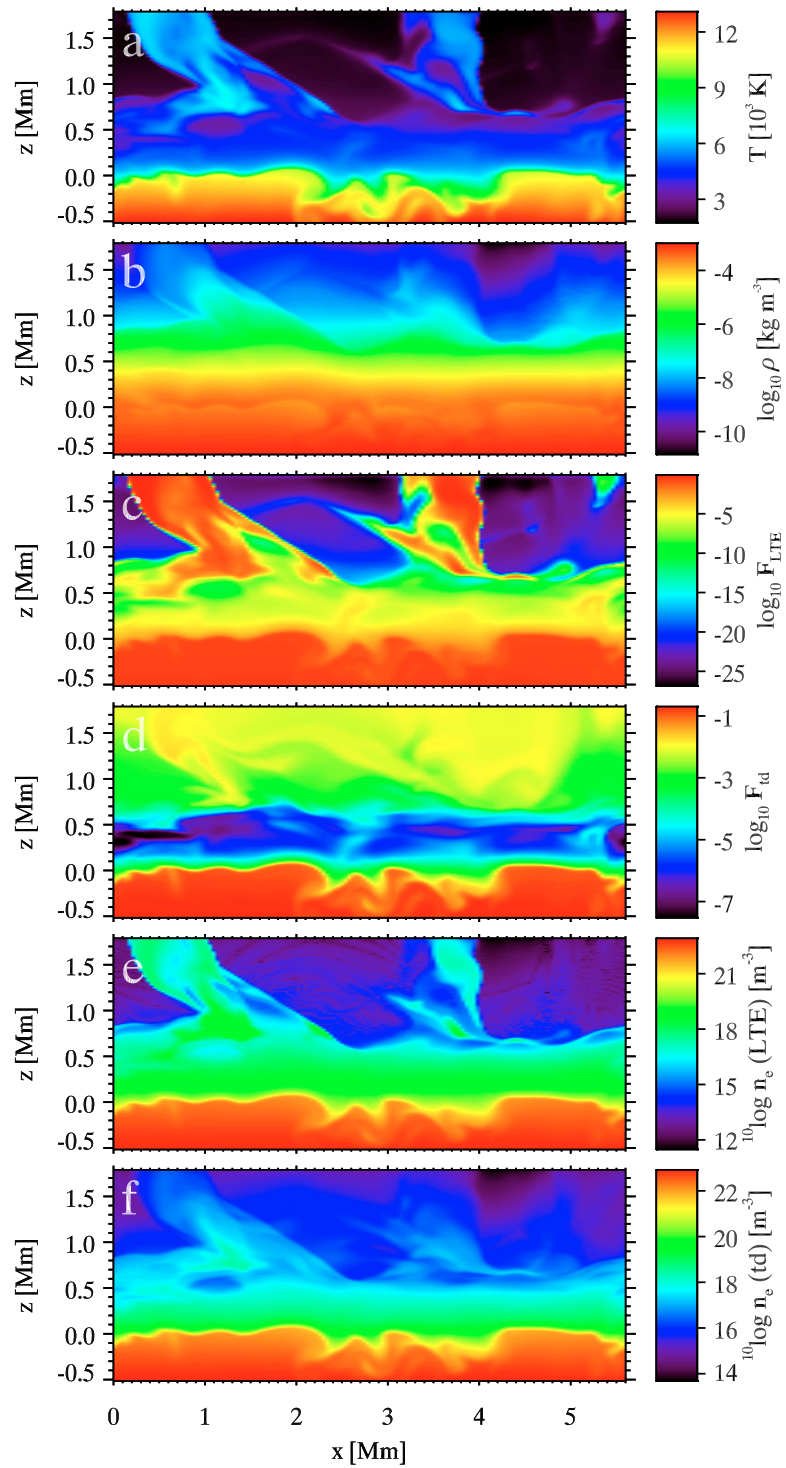

Fig. 3. Vertical cut through the snapshot along the line indicated in Fig. 2. a) gas temperature; b) mass density; LTE c) and TD-NLTE d) ionisation degree; LTE e) and TD-NLTE f) electron density. Note that the deepest layers of the model are not shown.

time-dependent ionisation on the equation of state, causing too low shock temperatures in our $\mathrm{CO}^{5} \mathrm{BOLD}$ model (Carlsson \& Stein 1992). Third, CS2002 include a transition region and corona in their model which influence the ionisation degree in the upper chromosphere, both by Lyman radiation ionising the upper chromosphere and the varying height of their transition zone. Their transition zone is sometimes considerably lower than 1.5 Mm, influencing the average ionisation degree. Fourth, it cannot be ascertained that the fixed radiative rates that we employ, which are accurate in $1 \mathrm{D}$, are also accurate in 3D. This might affect our results as well.

Contributions to the electron density from other elements than hydrogen are still treated under the assumption of LTE. This will mostly affect the upper photosphere where the hydrogen ionisation degree is so low that metals such as iron, magnesium, and silicon are the main electron donors. Hydrogen is the dominant donor in all other regions. However, in the chromospheric shocks the other elements contribute around $10 \%$ of the electrons (see Fig. 7). Thus we expect a small error in the chromospheric
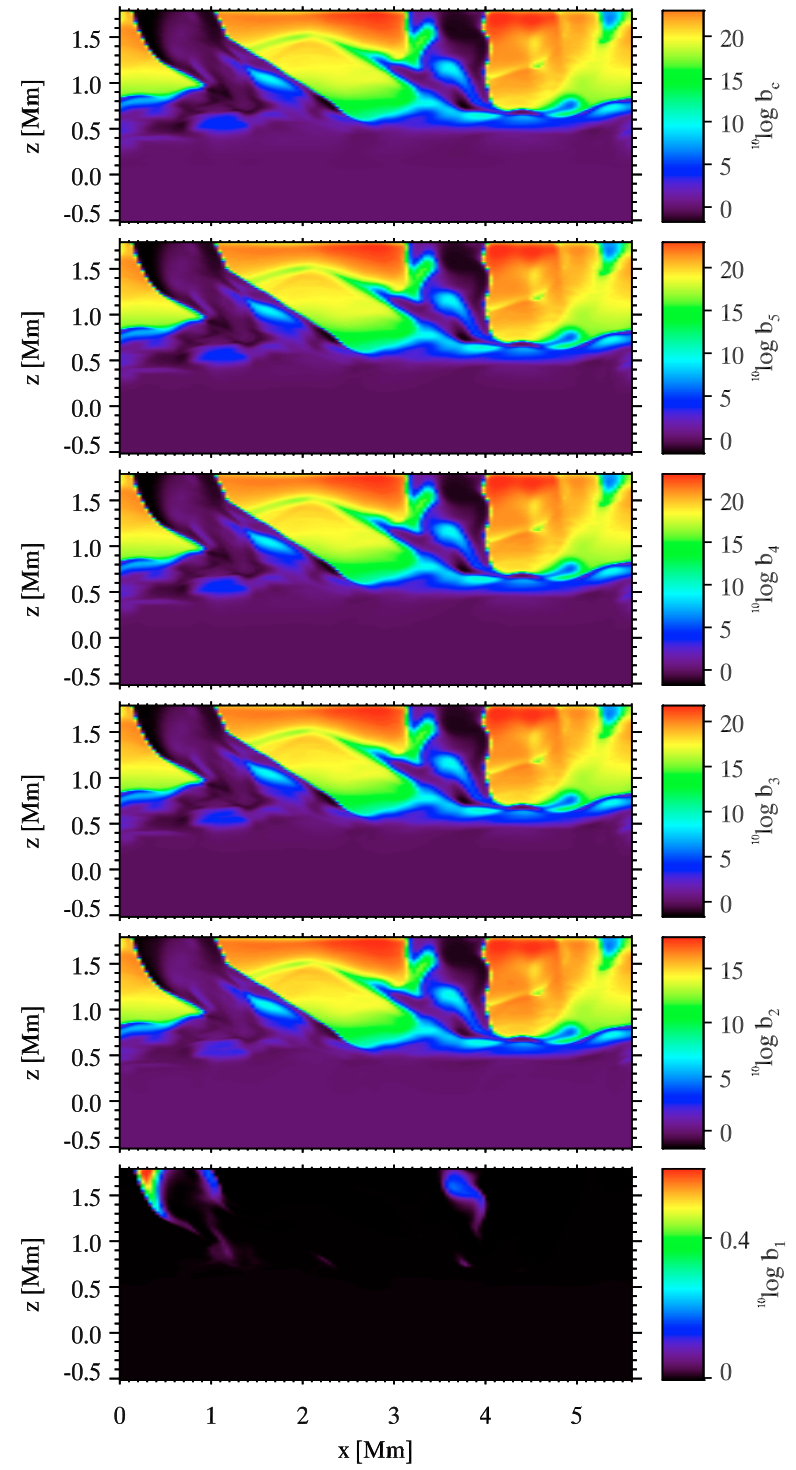

Fig. 4. Departure coefficients of our model hydrogen atom in the same cut as Fig. 3 for the continuum and level $n=5$ down to level $n=1$ from top to bottom. The solution is nearly in LTE from the bottom of the computational domain up to $0.3 \mathrm{Mm}$ above the average Rosseland optical depth unity. The largest deviations occur in cool chromospheric regions in between shocks, where, owing to the low temperature, the Saha-Boltzmann equation predicts a very low occupation fraction for all excited levels.

electron density due to the LTE treatment of the other elements (see Rammacher \& Ulmschneider 2003 for TD-NLTE effects on magnesium ionisation). For future simulations that take the transition region into account, a TD-NLTE treatment of helium might be of importance for the electron density as well, as the higher temperatures will lead to significant helium ionisation.

All in all we conclude that our 3D simulation with timedependent hydrogen ionisation produces reasonably realistic if probably somewhat too low - results for the ionisation degree and electron density, given the level of necessary simplification and resulting increase in computational speed.

With our method we can supply snapshots of 3D (M-)HD solar atmosphere simulations for detailed radiative transfer calculations containing time-dependent electron densities. Up until now most MHD simulations could only provide LTE electron 


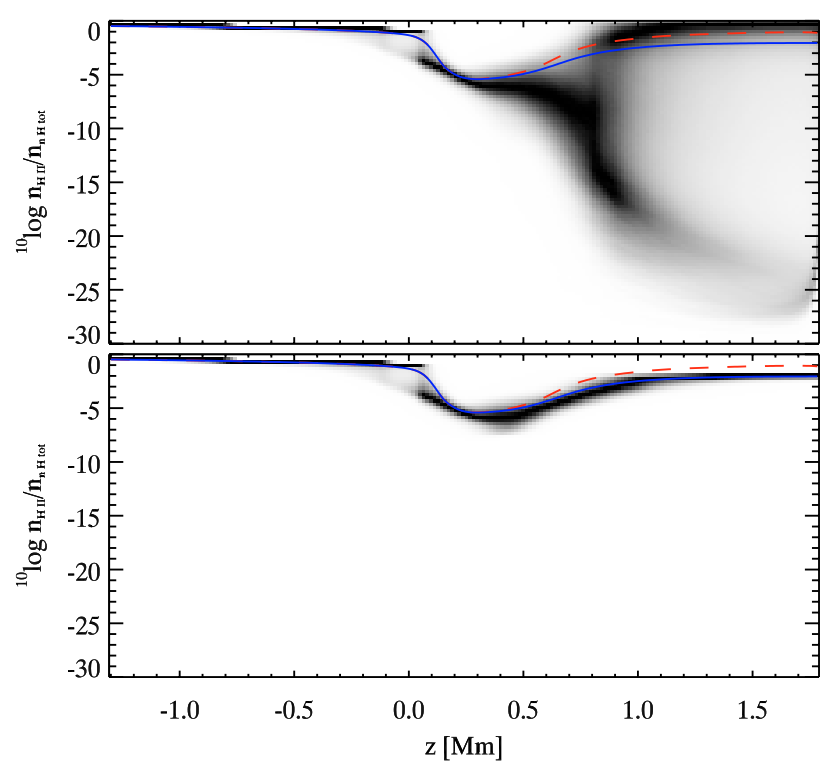

Fig. 5. Histogram of the logarithmic ionisation degree as a function of height for the LTE (upper panel) and TD-NLTE (lower panel) case. The averages in LTE and TD-NLTE are plotted as red dashed and blue solid lines, respectively. All columns have been individually scaled to maximum contrast to enhance visibility.

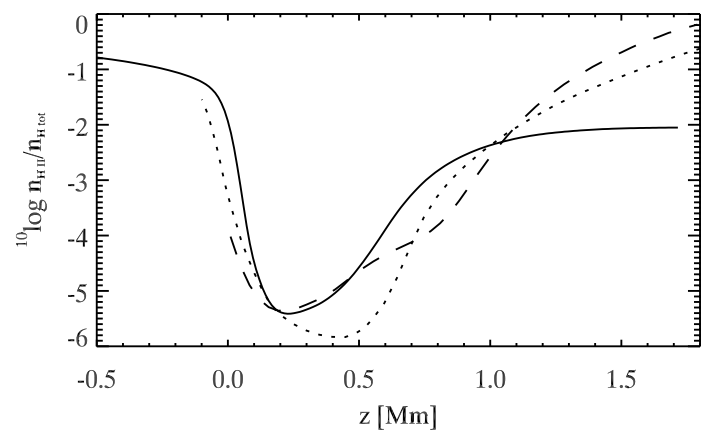

Fig. 6. Average TD-NLTE ionisation degree for our $\mathrm{CO}^{5} \mathrm{BOLD}$ model (solid), CS2002's RADYN model (dashed) and the statistical equilibrium FAL model $\mathrm{C}$ (dotted). The zero point of the height scale is the average $\tau_{500}=1$ height.

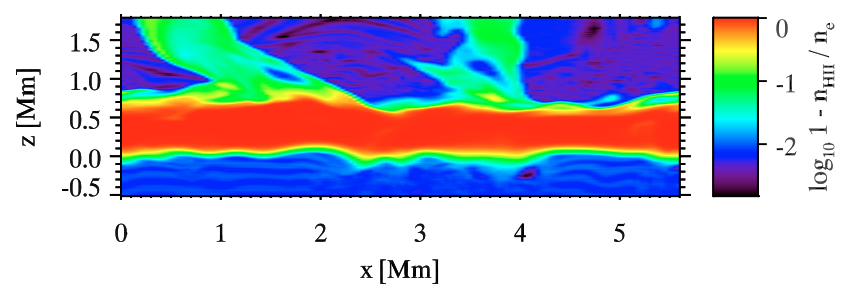

Fig. 7. Vertical cut through the snapshot along the line indicated in Fig. 2 showing the relative contribution of other elements than hydrogen to the electron density. In the chromosphere the other elements contribute mostly in the high temperature shocks.

densities, which are far from realistic in the chromosphere. The TD-NLTE electron densities are crucial in forward modelling of chromospheric diagnostics like the Ca II H\&K and infrared lines and (sub-)millimetre continua (see Leenaarts \& Wedemeyer-Böhm 2006 for an example of the effect on TD-NLTE electron densities on the latter), as collisions between electrons and atoms provide the coupling of the state of the gas to the radiation field via the source function, and affect the opacities of for example $\mathrm{H}$ free-free and $\mathrm{H}^{-}$bound-free and free-free transitions.

As it is now, the code computes the ionisation degree and electron density from the state parameters defined by the hydrodynamics simulation. Future improvement of the method should take into account the back-coupling of the ionisation to the equation of state. The deviation from instantaneous ionisation equilibrium makes it impossible to use pre-computed lookup tables for the equation of state. In addition, the lookup tables that give the bin-averaged source function and opacity (computed in LTE as function of temperature and pressure) are then no longer consistent with the state of the gas. New methods have to be developed to handle this complex problem. In particular, implementing such physics in a Riemann-solver as used in $\mathrm{CO}^{5} \mathrm{BOLD}$ is non-trivial. Nevertheless this work is currently in progress. Significant influence on chromospheric wave propagation and temperature structure in the chromosphere can be expected (cf. Carlsson \& Stein 1992).

Another necessary improvement is the relaxation of the assumption of the fixed radiative rates. This is mainly important for the Balmer continuum, which is the driver of the ionisation. A radiative rate that is proportional to the mean grey intensity, or, in the case of multi-group opacity methods, the mean intensity in the continuum is an obvious choice. Such a method would take the variation in the average radiation field due to the granulation pattern into account. It will give more accurate results in the case of strong horizontal inhomogeneities in gas temperature and density in the upper photosphere, as it is the case in the presence of magnetic fields.

Simulations for other stellar types than the Sun are possible but involve re-adjustment of the radiation temperatures. This requires a similar detailed 1D analysis and calibration as Sollum performed for the Sun for each spectral type separately.

Acknowledgements. The authors would like to thank O. Steiner, J. Bruls, and H.-G. Ludwig for illuminating discussions and R. J. Rutten, C. Keller and the referee for improvements to the manuscript. H. Uitenbroek provided his RH code. J. Leenaarts recognises support from the Deutscher Akademischer Austauschdienst, support from the USO-SP International Graduate School for Solar Physics (EU contract nr. MEST-CT-2005-020395) and hospitality at the Kiepenheuer-Institut für Sonnenphysik. S. Wedemeyer-Böhm was supported by the Deutsche Forschungsgemeinschaft (DFG), grant Ste 615/5.

\section{References}

Brown, P., Byrne, G., \& Hindmarsh, A. 1989, SIAM J. Sci. Stat. Comp., 10, 1038

Carlsson, M., \& Stein, R. F. 1992, ApJ, 397, L59

Carlsson, M., \& Stein, R. F. 1995, ApJ, 440, L29

Carlsson, M., \& Stein, R. F. 2002, ApJ, 572, 626

Fontenla, J. M., Avrett, E. H., \& Loeser, R. 1993, ApJ, 406, 319

Freytag, B., Steffen, M., \& Dorch, B. 2002, Astron. Nachr., 323, 213

Hansteen, V. 1993, ApJ, 402, 741

Johnson, L. C. 1972, ApJ, 174, 227

Kneer, F. 1980, A\&A, 87, 229

Leenaarts, J., \& Wedemeyer-Böhm, S. 2005, A\&A, 431, 687

Leenaarts, J., \& Wedemeyer-Böhm, S. 2006, in ASP Conf. Ser., in press

Rammacher, W., \& Ulmschneider, P. 2003, ApJ, 589, 988

Seaton, M. J. 1960, Reports of Progress in Physics, 23, 313

Sollum, E. 1999, Master's thesis, University of Oslo

Uitenbroek, H. 2001, ApJ, 557, 389

Ulmschneider, P., Rammacher, W., Musielak, Z. E., \& Kalkofen, W. 2005, ApJ, 631, L155

Vernazza, J. E., Avrett, E. H., \& Loeser, R. 1981, ApJS, 45, 635

Wedemeyer, S. 2003, Ph.D. Thesis, University of Kiel,

http://e-diss.uni-kiel.de/diss_764/

Wedemeyer, S., Freytag, B., Steffen, M., Ludwig, H.-G., \& Holweger, H. 2004, A\&A, 414, 1121 